\title{
Minimum Wiener Index Value and Polar Graph of Book Graphs
}

\author{
Fuyi Wei ${ }^{1, *}$, Yongyu Yang ${ }^{1}$, Meijun Liang ${ }^{1}, Q u W^{2}{ }^{2}$ \\ ${ }^{1}$ Institute of Mathematics and Information Software College, South China Agricultural University, Guangzhou, China \\ ${ }^{2}$ School of Accountancy, Central University of Finance and Economics, Beijing, China
}

\section{Email address:}

weifuyi@scau.edu.cn (Fuyi Wei), 271960884@qq.com (Yongyu Yang), 1152448561@qq.com (Meijun Liang)

*Corresponding author

\section{To cite this article:}

Fuyi Wei, Yongyu Yang, Meijun Liang, Qu Wei. Minimum Wiener Index Value and Polar Graph of Book Graphs. Science Discovery. Vol. 4, No. 4, 2016, pp. 213-219. doi: 10.11648/j.sd.20160404.12

Received: June 13, 2016; Accepted: June 14, 2016; Published: July 20, 2016

Abstract: Topological molecular index, which is widely applied in QSPR/QSAR studies of chemical theory, is one of the most active research fields in modern chemical graph theory. Wiener index is the sum of the distance between any two points in the graph. In this paper, we use the method of graph theory, we define three transformations, and prove the six lemma, get minimum value of the wiener index of book graphs, and determines the book graph with smallest wiener index.

Keywords: Book Graph, Wiener Index, Minimum Values, Polar Diagram

\section{书图的维纳指数的极小值与极图}

\author{
魏福义 ${ }^{1 *}$, 杨泳愉 ${ }^{1}$, 梁美君 ${ }^{1}$, 魏趣 $^{2}$ \\ '数学与信息学院, 华南农业大学, 广州, 中国 \\ ${ }^{2}$ 会计学院, 中央财经大学, 北京, 中国 \\ 邮箱 \\ weifuyi@scau. edu.cn（魏福义），271960884@qq. com（杨泳愉），1152448561@qq. com（梁美君）
}

摘要: 分子拓扑指数被广泛地应用于理论化学的定量结构性质和活性关系的研究中, 图中任意两点间的距离之和称为 维纳指数。本文运用图论的方法, 定义了三种变换, 证明了六个引理, 得到了书图的维纳指数的最小值, 并且确定了 维纳指数达到最小的极图。

关键词: 书图, 维纳指数, 极小值, 极图

\section{1. 引言}

在一个无向图 $G(V, E)$ 中，任意两个不同顶点 $u, v$ 都 有路径相连, 则图 $G$ 称为连通图; 顶点 $u, v$ 之间的最短路 的长度称为 $u, v$ 之间的距离, 记为 $d(u, v)$; 与顶点 $u$ 关
联的边数称为 $u$ 的度, 记为 $d(u)$, 度为 1 的顶点称为悬挂 点; 没有圈的简单连通无向图称为树。一个顶点数大于或 者等于 3 的含圈连通无向图 $G_{1}$, 其含有唯一的一条边 $e$, 满足其任意的两个诱导圈的公共边恰好是边 $e$, 则图 $G_{1}$ 称 为书图 (见图1, 其中虚线圆形是以圈上点为根点的树)。 


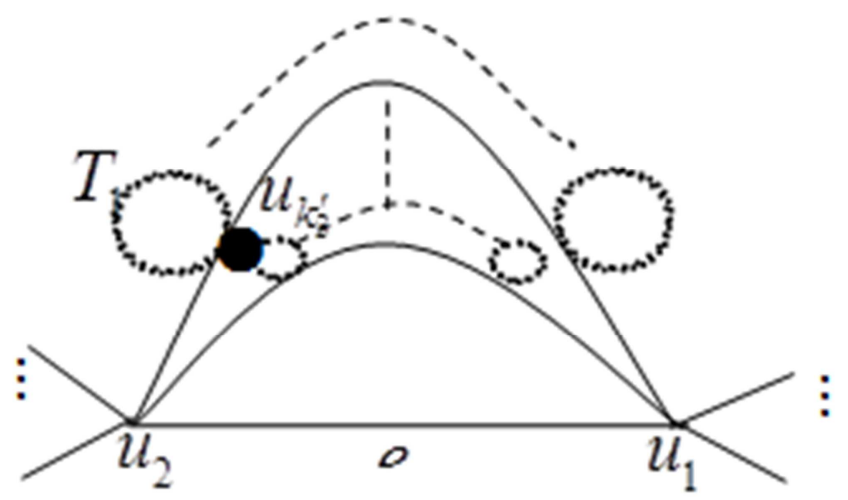

图1书图 $G_{1}$ 。

边 $e$ 的两个顶点 $u_{1} 、 u_{2}$ 称为 $G_{1}$ 的公共顶点, 图上的 其它非悬挂的顶点称为非公共顶点。书图 $G_{1}$ 删除它的全 部树 (保留根点) 所得的图象一本书, 边 $e$ 为书背, 每个 圈和以圈上的点为根点的树构成的图称为书的 “页” 图。 设图 $D\left(V_{1}, E_{1}\right)$ 和 $H\left(V_{2}, E_{2}\right)$ 为两个连通无向图, 若存 在双映射 $f: V_{1} \rightarrow V_{2}, \forall\left(v_{i}, v_{j}\right) \in E_{1}$ ，当且仅当 $\left(f\left(v_{i}\right), f\left(v_{j}\right)\right) \in E_{2}$, 则图 $D\left(V_{1}, E_{1}\right)$ 和 $H\left(V_{2}, E_{2}\right)$ 称 为同构, 记为 $D \cong H$ 。用 $d_{D}(u, v)$ 和 $d_{H}(u, v)$ 分别表示 顶点 $u, v$ 在图 $D$ 和图 $H$ 中的距离。图 $G$ 的顶点集和边集 分别为 $V(G), E(G), G$ 的顶点数和边数分别用 $|V(G)|$ 和 $|E(G)|$ 表示。若 $|V(G)|<+\infty$, 图 $G$ 为有限图。本文 只研究有限连通书图。

图 $G$ 的维纳指数定义为图中任意两点间的距离之和, 记为 $W(G)$, 即

$$
W(G)=\sum_{u, v \in V(G)} d(u, v)
$$

维纳指数是在研究化学分子结构模型中提出的拓扑 指数之一 [1-4], 并已被证实能够揭示物质中分子之间的 性能关系。文献 [5-6] 中综述了维纳指数近五十年的研究 成果。文献 [7]中给出了书图的相关概念。本文研究了书 图的维纳指数的最小值, 并确定了达到最小的维纳指数的 极图。

\section{2. 主要定理}

对于任意的书图, 可以通过三种变换得到达到最小维 纳指数的极图。

变换 $A$ : 把图 $G_{1}$ 中任意一棵树变成以原来圈上根点 为中心点的同阶星图, 其它结构不变。

设书图 $G_{1}$ 通过变换 $A$ 后得到的图为 $G_{1}^{\prime}$ (见图2), 记为 $A\left(G_{1}\right)=G_{1}^{\prime}$ 。

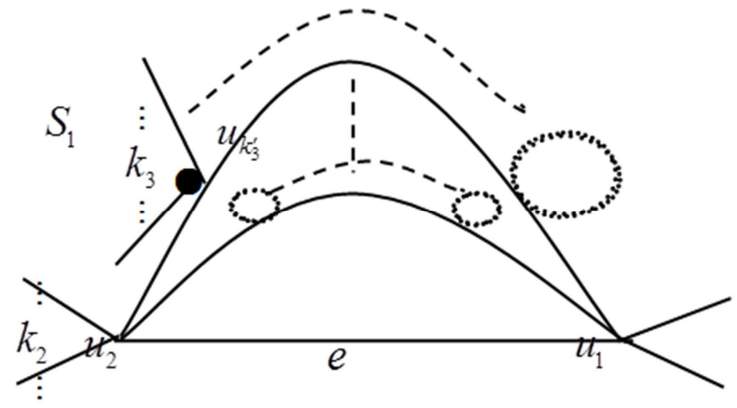

图2书图 $G_{1}^{\prime}$ 。

性质2. 1 设 $A\left(G_{1}\right)=G_{1}^{\prime}$, 则 $W\left(G_{1}\right) \geq W\left(G_{1}^{\prime}\right)$, 等 式成立当且仅当 $G_{1} \cong G_{1}^{\prime}$ 。

证明不妨设 $G_{1}$ 中的树 $T_{1}$ 的阶为 $k_{3}+1$ (含根点), 变换 $A$ 把图 $G_{1}$ 中的树 $T_{1}$ 变成同阶星图 $S_{1}$ 所得图记为 $G_{1}^{\prime}$ (见图2）。

对于顶点 $u, v \in V\left(G_{1}\right)$, 及对应顶点 $u^{\prime}, v^{\prime} \in V\left(G_{1}^{\prime}\right)$, 当 $u, v$ 至 少有一个不属于 $V\left(T_{1}\right)$ 时， $d_{G}(u, v) \geq d_{G^{\prime}}\left(u^{\prime}, v^{\prime}\right)$, 等式成立当且仅当 $u, v$ 都不属于 $V\left(T_{1}\right)$, 或属于 $V\left(T_{1}\right)$ 的顶点与根点相邻。

当 $u, v$ 都属于 $V\left(T_{1}\right)$ 时,

$$
\begin{array}{r}
\sum_{u, v \in V\left(T_{1}\right)} d(u, v)=\sum_{(u, v) \in E\left(T_{1}\right)} d(u, v)+\sum_{(u, v) \notin E\left(T_{1}\right)} d(u, v) \\
=k_{3}+\sum_{(u, v) \notin E\left(T_{1}\right)} d(u, v) \geq k_{3}+2\left[C_{k_{3}+1}^{2}-k_{3}\right]
\end{array}
$$

等式成立当且仅当 $T_{1}$ 中不相邻的顶点的距离为 2 , 即 $T_{1}$ 为星图（见图2和图3）。

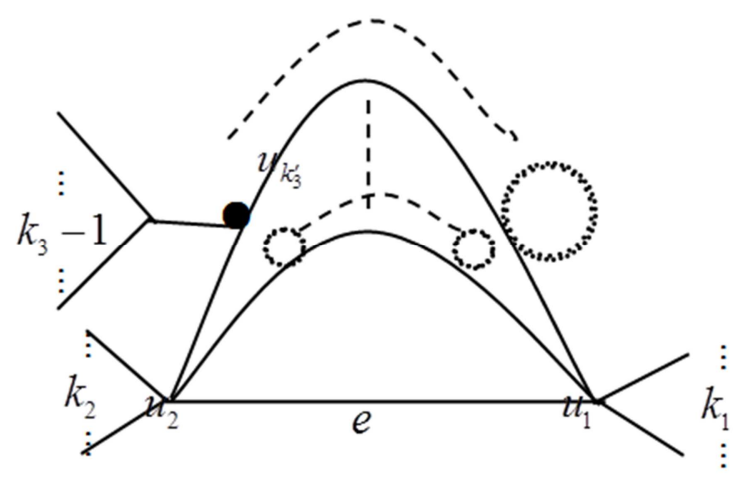

图3 书图 $G_{1}^{\prime \prime}$ 。

直接计算: $W\left(G_{1}^{\prime \prime}\right) \geq W\left(G_{1}^{\prime}\right)$, 等式成立当且仅当 $k_{3}=1$, 此时 $G_{1}^{\prime} \cong G_{1}^{\prime \prime}$ 。

由上述讨论知: $W\left(G_{1}\right) \geq W\left(G_{1}^{\prime}\right)$, 等式成立当且仅 当 $G_{1} \cong G_{1}^{\prime}$ 。证毕。 
不妨设运用变换 $A$ “ $r$ ” 次, 可以把图 $G_{1}$ 中树都分 别变换为以圈上根点为中心点的同阶星图, 即把图 $G_{1}$ 变 换成图 $G_{2}$, 记为 $A^{(r)}\left(G_{1}\right)=G_{2}$ （如图4）。

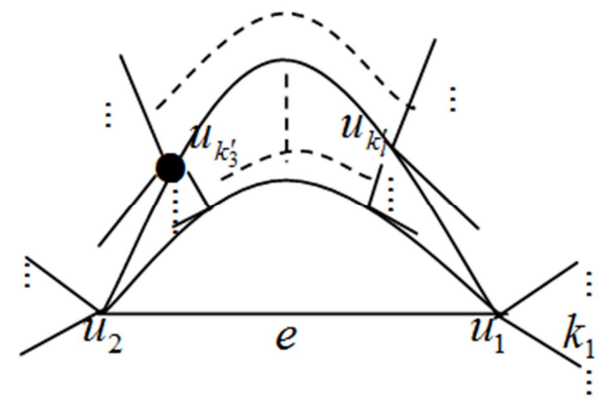

图4书图 $G_{2}$ 。

\section{由性质2.1可知:}

引理2. 1 设 $A^{(r)}\left(G_{1}\right)=G_{2}$, 则 $W\left(G_{1}\right) \geq W\left(G_{2}\right)$, 等式成立当且仅当 $G_{1} \cong G_{2}$ 。

任意取 $G_{2}$ 中非公共顶点含星图的一页, 记为 $U_{1}$ 。页 $U_{1}$ 是 $G_{2}$ 的一个单圈子图。圈长为 $r$, 圈上非公共顶点 $u_{k_{i}^{\prime}}$ 上的悬挂点个数为 $k_{i}>0(i=3,4, \ldots, l)$, $d\left(u_{2}, u_{k_{3}^{\prime}}\right)=s （ 见$ 图5）。

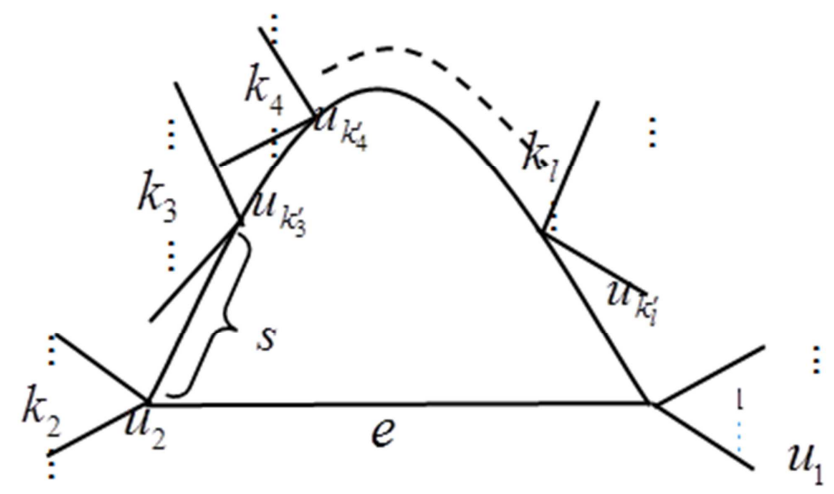

图5 书图。

令: $V_{1}=\left\{u \in V\left(U_{1}\right) \mid d(u)=1\right\} ; V_{2}=V\left(U_{1}\right) / V_{1}$;

$$
\begin{gathered}
V_{3}=V\left(G_{2}\right) / V\left(U_{1}\right) ; \\
V_{4}=\left\{u \in V_{1} \mid d\left(u, u_{1}\right)=1 \text { 或 } d\left(u, u_{2}\right)=1\right\} ; \\
V_{5}=\left\{u \in V_{1} / V_{4} \mid d\left(u, u_{2}\right) \leq d\left(u, u_{1}\right)\right\} ; \\
V_{6}=\left\{u \in V_{1} / V_{4} \mid d\left(u, u_{2}\right)>d\left(u, u_{1}\right)\right\} 。
\end{gathered}
$$

分别记 $\left|V_{2}\right|=r,\left|V_{3}\right|=q,\left|V_{4}\right|=m,\left|V_{5}\right|=i,\left|V_{6}\right|=j$ 。圈 $U_{1}$ 上所有非公共顶点的悬挂点保持原有的距离按以下规
则作定向移动: 若 $i \geq j$, 把该圈上所有非公共顶点的悬 挂点保持原来的距离沿逆时针方向移动, 直至距离 $u_{2}$ 最 近的的非公共顶点 $u_{k_{3}^{\prime}}$ 上的悬挂点移到 $u_{2}$ 上（圈中点不 动）; 若 $i<j$, 把该圈上所有非公共顶点的悬挂点保持 原来的距离沿顺时针方向移动, 直至距离 $u_{1}$ 最近的的非 公共顶点 $u_{k_{1}^{\prime}}$ 上的悬挂点移到 $u_{1}$ 上（圈中点不动）。以上 两种移动称为有向移动。

变换 $B$ : 把书图 $G_{2}$ 中某 “页” 进行一次有向移动后 的图为 $G_{2}^{\prime}$ (当 $i \geq j$ 时) 或 $\tilde{G}_{2}^{\prime}$ (当 $i<j$ 时), 记为

$$
B\left(G_{2}\right)=G_{2}^{\prime} \text { （见图6）或 } B\left(G_{2}\right)=\widetilde{G}_{2}^{\prime} \text { 。 }
$$

性质2. 2 设 $B\left(G_{2}\right)=G_{2}^{\prime}$, 则 $W\left(G_{2}\right) \geq W\left(G_{2}^{\prime}\right)$, 等 号成立当且仅当 $i=j$ 或 $m+q=0$; 若 $B\left(G_{2}\right)=\widetilde{G}_{2}^{\prime}$, 则 $W\left(G_{2}\right)>W\left(G_{2}^{\prime}\right)$ 。

证明 若 $B\left(G_{2}\right)=G_{2}^{\prime}$, 由定向移动规则可知 $i \geq j$, 则把该圈上所有非公共顶点的悬挂点沿逆时针方向移动 直至距离 $u_{2}$ 最近的的非公共顶点 $u_{k_{3}^{\prime}}$ 上的悬挂点移到 $u_{2}$ 上（圈中点不动）, 设此时移动了 $S$ 步（见图6）。

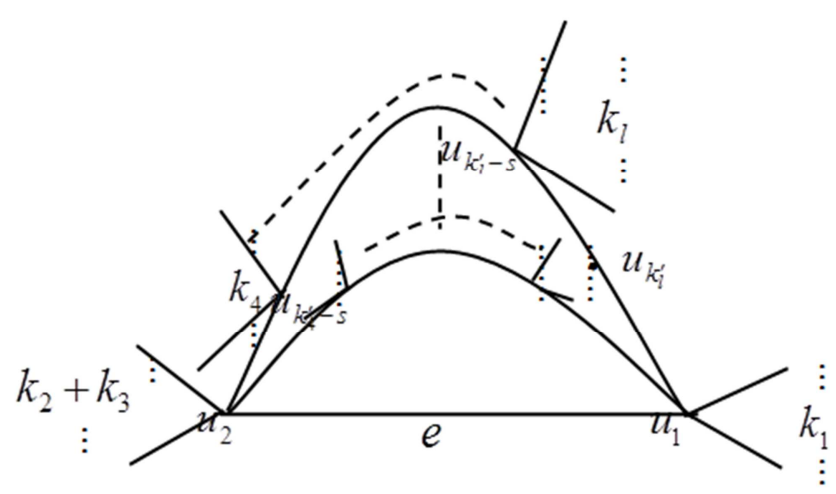

图6 书图 $G_{2}^{\prime}$ 。

$\forall u, v \in V\left(G_{2}\right)$ 及对应点 $u^{\prime}, v^{\prime} \in V\left(G_{2}^{\prime}\right)$, 有:

(1) $\sum_{u, v \in V_{k}(k=2,3)} d_{G_{2}}(u, v)-\sum_{u^{\prime}, v^{\prime} \in V_{k}^{\prime}(k=2,3)} d_{G_{2}^{\prime}}\left(u^{\prime}, v^{\prime}\right)=0$;

(2) $\sum_{u, v \in V_{1}} d_{G_{2}}(u, v)-\sum_{u^{\prime}, v^{\prime} \in V_{1}^{\prime}} d_{G_{2}^{\prime}}\left(u^{\prime}, v^{\prime}\right) \geq(i-j) \cdot s \cdot m$ 等号成立当且仅当 $i=j$ 或 $m=0$;

(3) $\sum_{u \in V_{1}, v \in V_{2}} d_{G_{2}}(u, v)-\sum_{u^{\prime} \in V_{1}^{\prime}, v^{\prime} \in V_{2}^{\prime}} d_{G_{2}^{\prime}}\left(u^{\prime}, v^{\prime}\right)=0$;

(4) $\sum_{u \in V_{1}, v \in V_{3}} d_{G_{2}}(u, v)-\sum_{u^{\prime} \in V_{1}^{\prime}, v^{\prime} \in V_{3}^{\prime}} d_{G_{2}^{\prime}}\left(u^{\prime}, v^{\prime}\right) \geq(i-j) \cdot s \cdot q$ 等号成立当且仅当 $i=j$ 或 $q=0$; 
(5)

$\sum_{u \in V_{2}, v \in V_{3}} d_{G_{2}}(u, v)-\sum_{u^{\prime} \in V_{2}^{\prime}, v^{\prime} \in V_{3}^{\prime}} d_{G_{2}^{\prime}}\left(u^{\prime}, v^{\prime}\right)=0$ 。

由以上讨论知: $\begin{array}{r}W\left(G_{2}\right)-W\left(G_{2}^{\prime}\right) \geq(i-j) s \cdot m+(i-j) s \cdot q \\ =(i-j) s \cdot(m+q)\end{array}$

即 $W\left(G_{2}\right) \geq W\left(G_{2}^{\prime}\right)$ 等号成立当且仅当 $i=j$ 或 $m+q=0$ 。

同理可证: 若 $B\left(G_{2}\right)=\tilde{G}_{2}{ }^{\prime}$, 则 $W\left(G_{2}\right)>W\left(G_{2}^{\prime}\right)$ 证毕。

不妨设运用变换 $B$ “ $y$ ” 次, 把图 $G_{2}$ 中非公共顶点 上的悬挂点都移动到公共顶点上, 即把 $G_{2}$ 变换成 $G_{3}$ （见 图7）, 记为 $B^{(y)}\left(G_{2}\right)=G_{3}$ 。

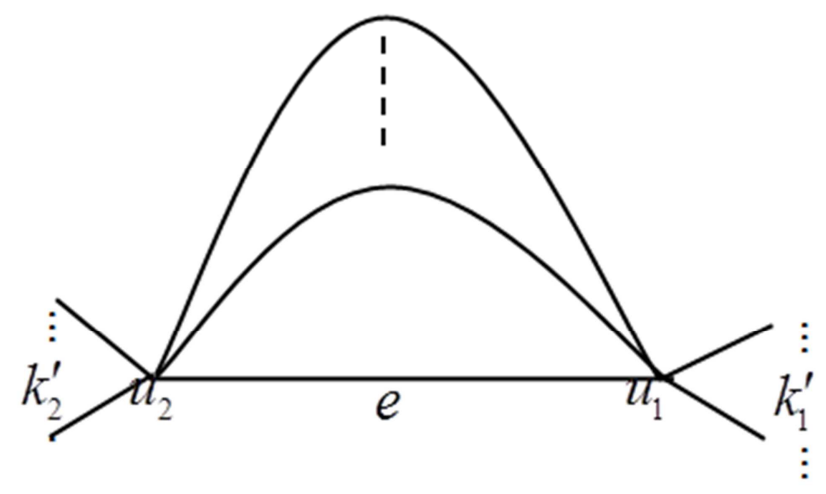

图7 书图 $G_{3}$ 。

引理2. 2 设 $B^{(y)}\left(G_{2}\right)=G_{3}$, 则 $W\left(G_{2}\right)>W\left(G_{3}\right)$ 。

证明由性质 2.2 知: $W\left(G_{2}\right) \geq W\left(G_{2}^{\prime}\right)$, 分情况讨 论不等式成立条件如下:

(1) $m+q=0$ (即 $G_{2} \cong U_{1}$ ），只有一个或两个相 邻的非公共顶点有悬挂点时, 即

$G_{2} \cong G_{3}, W\left(G_{2}\right)=W\left(G_{3}\right)$; 当有两个不相邻或 三个及以上非公共顶点上有悬挂点时, 通过变换 $B y$ 次 把悬挂点移动到公共顶点后, 不满足 $m+q=0$, 故 $W\left(G_{2}\right)>W\left(G_{3}\right) ;$

(2) 当所有页上的悬挂点都满足 $i=j=0$, 即 $G_{2} \cong G_{3}, W\left(G_{2}\right)=W\left(G_{3}\right)$; 当 $i=j \neq 0$ 时通过有限次 变换 $B$ 总会使得 $i \neq j$, 因此 $W\left(G_{2}\right)>W\left(G_{3}\right)$ 。

综上所述: 当 $G_{2} \cong G_{3}, W\left(G_{2}\right)=W\left(G_{3}\right)$; 其余情 况下 $B^{(y)}\left(G_{2}\right)=G_{3}$, 得 $W\left(G_{2}\right)>W\left(G_{3}\right)$ 。证毕。

定义: 把图 $G_{3}$ 中 $u_{1}$ 上的全部悬挂点移动到 $u_{2}$ 上所 得的图记为 $G_{4}$ （见图8）。

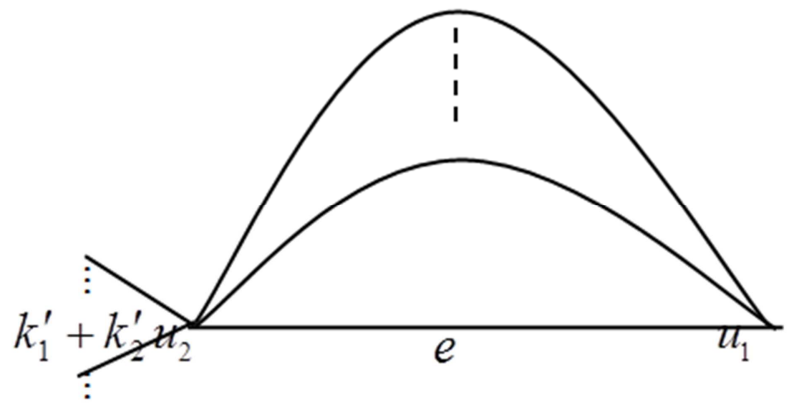

图8 书图 $G_{4}$ 。

引理 $2.3 W\left(G_{3}\right) \geq W\left(G_{4}\right)$, 等式成立当且仅当 $G_{3} \cong G_{4}$ 。

证明

$W\left(G_{3}\right)-W\left(G_{4}\right)=3 k_{1}^{\prime} \cdot k_{2}^{\prime}-2 k_{1}^{\prime} \cdot k_{2}^{\prime}=k_{1}^{\prime} \cdot k_{2}^{\prime} \geq 0$, $W\left(G_{3}\right) \geq W\left(G_{4}\right)$, 等式成立当且仅当 $k_{1}^{\prime}=0$ 或 $k_{2}^{\prime}=0$, 即 $G_{3} \cong G_{4}$, 证毕。

任意取 $G_{4}$ 中长为 $l(l>3)$ 的一个圈和顶点 $u_{2}$ 的悬 挂边构成的图记为 $U_{2}$ （见图9）。

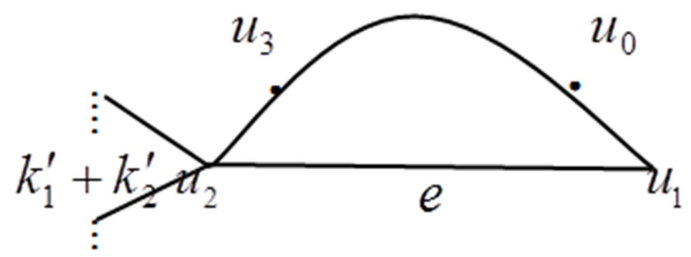

图9 页图 $U_{2}$ 。

变换 $C$ : 把 $G_{4}$ 内的页图 $U_{2}$ 换成长为 3 的一个圈, 且 在 $u_{2}$ 点增加 $(l-3)$ 个悬挂点, 其余部分不变所得的图为 $G_{4}^{\prime}$ （见图10）, 记为 $C\left(G_{4}\right)=G_{4}^{\prime}$ 。

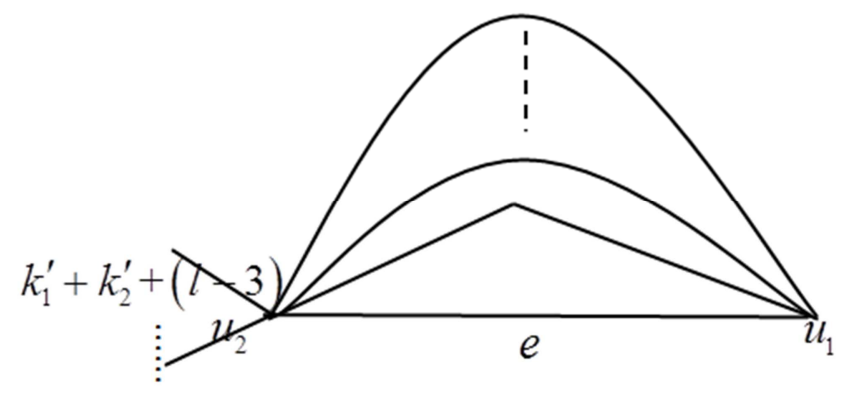

图10 页图 $G_{4}^{\prime}$ 。

性质 2.4 设图 $U_{2}$ 为图 $G_{4}$ 的一个页图, $C\left(G_{4}\right)=G_{4}^{\prime}$, 则 $W\left(G_{4}\right) \geq W\left(G_{4}^{\prime}\right)$, 等式成立当且仅当 $k_{1}^{\prime}+k_{2}^{\prime}=0$ 且 $U_{2}$ 的圈长为 4 或者 $G_{4} \cong C_{5}, C_{5}$ 表示圈长为 5 的单圈图。 
证明 设 $u_{3}$ 和 $u_{0}$ 分别表示页图 $U_{2}$ 中与 $u_{2}, u_{1}$ 相邻的 非公共顶点,

$$
H=\left\{u \in U_{2} \mid d\left(u, u_{1}\right) \geq 2, d\left(u, u_{2}\right) \geq 2\right\},
$$

$$
J=G_{4} /\left\{\left(u_{3}, u_{0}\right) \cup H\right\},
$$

$\forall u, v \in V\left(G_{4}\right)$ 及对应点 $u^{\prime}, v^{\prime} \in V\left(G_{4}^{\prime}\right)$, 分情况讨 论:

（1）当 $|H|=0$, 即 $U_{2}$ 的圈长为 4 。

由

$$
C\left(G_{4}\right)=G_{4}^{\prime}
$$

$E\left(G_{4}^{\prime}\right)=E\left(G_{4}^{\prime}\right) \bigcup\left\{\left(u_{2}, u_{0}\right),\left(u_{1}, u_{3}\right)\right\} /\left\{\left(u_{0}, u_{1}\right),\left(u_{0}, u_{3}\right)\right\}$ 则 $W\left(G_{4}\right)-W\left(G_{4}^{\prime}\right)=k_{1}^{\prime}+k_{2}^{\prime} \geq 0$

故 $W\left(G_{4}\right) \geq W\left(G_{4}^{\prime}\right)$

等式成立当且仅当 $k_{1}^{\prime}+k_{2}^{\prime}=0$ 且 $U_{2}$ 的圈长为 4 。

（2）当 $|H|>0$, 不妨设 $|H|=x,|J|=p \geq 2$, 分 情况讨论:

(2.1) $u, v \in H$

$$
\begin{aligned}
& \sum_{u, v \in H} d_{G_{4}}(u, v)-\sum_{u^{\prime}, v^{\prime} \in H^{\prime}} d_{G_{4}^{\prime}}\left(u^{\prime}, v^{\prime}\right) \\
& =\frac{x(x-1)(x+1)}{6}-x(x-1)=\frac{x(x-1)(x-5)}{6}
\end{aligned}
$$

(2.2) $u \in H, v \in\left\{u_{0}, u_{3}\right\}$

$$
\begin{aligned}
& \sum_{u \in H, v \in\left\{u_{0}, u_{3}\right\}} d_{G_{4}}(u, v)-\sum_{u^{\prime} \in H^{\prime}, v^{\prime} \in\left\{u_{0}^{\prime}, u_{3}^{\prime}\right\}} d_{G_{4}^{\prime}}\left(u^{\prime}, v^{\prime}\right) \\
= & \left\{\begin{array}{l}
x(x-3), x \leq 4 \\
{\left[\sum_{i=5}^{x}\left(2+\left\lfloor\frac{i}{2}\right\rfloor\right)+10-2 x\right\rfloor \times 2, x>4}
\end{array}\right.
\end{aligned}
$$

(2.3) $u \in H, v \in J$

$$
\sum_{u \in H, v \in J} d_{G_{4}}(u, v)-\sum_{u^{\prime} \in H^{\prime}, v^{\prime} \in J^{\prime}} d_{G^{\prime}}\left(u^{\prime}, v^{\prime}\right) \geq x+x\left\lceil\frac{p+\left(k_{1}^{\prime}+k_{2}^{\prime}\right)}{2}\right\rceil-1
$$

$u, v \notin H$ 时, 分四种情况讨论:

(2. 4. 1) $d_{G_{4}}\left(u_{0}, u_{3}\right)-d_{G_{4}^{\prime}}\left(u_{0}^{\prime}, u_{3}^{\prime}\right)=\left\{\begin{array}{l}0, x=1 \\ 1, x>1\end{array} ;\right.$

(2.4.2) $\sum_{u, v \in J} d_{G_{4}}(u, v)-\sum_{u^{\prime}, v^{\prime} \in J^{\prime}} d_{G_{4}^{\prime}}\left(u^{\prime}, v^{\prime}\right)=0$;

(2. 4.3) $\sum_{v \in J} d_{G_{4}}\left(u_{0}, v\right)-\sum_{v^{\prime} \in J^{\prime}} d_{G_{4}^{\prime}}\left(u_{0}^{\prime}, v^{\prime}\right)=k_{1}^{\prime}+k_{2}^{\prime}$;

(2.4.4) $\sum_{v \in J} d_{G_{4}}\left(u_{3}, v\right)-\sum_{v^{\prime} \in J^{\prime}} d_{G_{4}^{\prime}}\left(u_{3}^{\prime}, v^{\prime}\right)=\left\lfloor\frac{p-\left(k_{1}^{\prime}+k_{2}^{\prime}\right)}{2}\right\rfloor 。$

把上述各式相加，可得：
当 $x=1, p=2, k_{1}^{\prime}+k_{2}^{\prime}=0 \quad$ 时 ， $W\left(G_{4}\right)-W\left(G_{4}^{\prime}\right)=0$, 即 $G_{4} \cong C_{5}$; 当 $x=1$ 且 $p>2$ 或 $x>1$ 时, $W\left(G_{4}\right)-W\left(G_{4}^{\prime}\right)>0$ 。

综上所述: $W\left(G_{4}\right) \geq W\left(G_{4}^{\prime}\right)$, 等式成立当且仅当 $k_{1}^{\prime}+k_{2}^{\prime}=0$ 且 $U_{2}$ 的圈长为 4 或者 $G_{4} \cong C_{5}$ 。证毕。

不妨设图 $G_{4}$ 中有 $f$ 个圈长度大于 3 , 则对图 $G_{4}$ 运用 变换 $C$ 共 $f$ 次, 得到图 $G_{5}$ (见图 11) , 记为 $C^{(f)}\left(G_{4}\right)=G_{5}$ 。

由性质2.4可得:

引理2.4 设 $C^{(f)}\left(G_{4}\right)=G_{5}$, 则 $W\left(G_{4}\right) \geq W\left(G_{5}\right)$, 等式成立当且仅当 $G_{4} \cong G_{5}$ 或者 $f=1, k_{1}^{\prime}+k_{2}^{\prime}=0$, $U_{2}$ 圈长为 4 或者 $G_{4} \cong C_{5} C_{5}$, 表示圈长为 5 的单圈图。

证明 设 $u_{3}$ 和 $u_{0}$ 分别表示页图 $U_{2}$ 中与 $u_{2}, u_{1}$ 相邻的 非公共顶点,

$$
H=\left\{u \in U_{2} \mid d\left(u, u_{1}\right) \geq 2, d\left(u, u_{2}\right) \geq 2\right\},
$$

$$
J=G_{4} /\left\{\left(u_{3}, u_{0}\right) \cup H\right\} .
$$

$\forall u, v \in V\left(G_{4}\right)$ 及对应点 $u^{\prime}, v^{\prime} \in V\left(G_{4}^{\prime}\right)$, 分情况讨 论:

（1）当 $|H|=0$, 即 $U_{2}$ 的圈长为 4 。

由

$$
C\left(G_{4}\right)=G_{4}^{\prime}
$$
$E\left(G_{4}^{\prime}\right)=E\left(G_{4}^{\prime}\right) \bigcup\left\{\left(u_{2}, u_{0}\right),\left(u_{1}, u_{3}\right)\right\} /\left\{\left(u_{0}, u_{1}\right),\left(u_{0}, u_{3}\right)\right\}$, 则 $W\left(G_{4}\right)-W\left(G_{4}^{\prime}\right)=k_{1}^{\prime}+k_{2}^{\prime} \geq 0$, 故 $W\left(G_{4}\right) \geq W\left(G_{4}^{\prime}\right)$, 等式成立当且仅当 $k_{1}^{\prime}+k_{2}^{\prime}=0$ 且 $U_{2}$ 的圈长为 4 。

（2）当 $|H|>0$, 不妨设 $|H|=x,|J|=p \geq 2$, 分 情况

讨论:

(2.1) $u, v \in H$

$$
\begin{aligned}
& \sum_{u, v \in H} d_{G_{4}}(u, v)-\sum_{u^{\prime}, v^{\prime} \in H^{\prime}} d_{G_{4}^{\prime}}\left(u^{\prime}, v^{\prime}\right) \\
= & \frac{x(x-1)(x+1)}{6}-x(x-1)=\frac{x(x-1)(x-5)}{6}
\end{aligned}
$$

(2.2) $u \in H, v \in\left\{u_{0}, u_{3}\right\}$

$$
\begin{aligned}
& \sum_{u \in H, v \in J} d_{G_{4}}(u, v)-\sum_{u^{\prime} \in H^{\prime}, v^{\prime} \in J^{\prime}} d_{G_{4}^{\prime}}\left(u^{\prime}, v^{\prime}\right) \\
\geq & x+x\left\lceil\frac{p+\left(k_{1}^{\prime}+k_{2}^{\prime}\right)}{2}\right\rceil-1
\end{aligned}
$$

（2.4） $u, v \notin H$ 时，分四种情况讨论: 
(2.4.1) $d_{G_{4}}\left(u_{0}, u_{3}\right)-d_{G_{4}^{\prime}}\left(u_{0}^{\prime}, u_{3}^{\prime}\right)=\left\{\begin{array}{l}0, x=1 \\ 1, x>1\end{array}\right.$;

(2.4.2) $\sum_{u, v \in J} d_{G_{4}}(u, v)-\sum_{u^{\prime}, v^{\prime} \in J^{\prime}} d_{G_{4}^{\prime}}\left(u^{\prime}, v^{\prime}\right)=0$;

(2. 4.3) $\sum_{v \in J} d_{G_{4}}\left(u_{0}, v\right)-\sum_{v^{\prime} \in J^{\prime}} d_{G_{4}^{\prime}}\left(u_{0}^{\prime}, v^{\prime}\right)=k_{1}^{\prime}+k_{2}^{\prime}$;

$\sum_{v \in J} d_{G_{4}}\left(u_{3}, v\right)-\sum_{v^{\prime} \in J^{\prime}} d_{G_{4}^{\prime}}\left(u_{3}^{\prime}, v^{\prime}\right)=\left\lfloor\frac{p-\left(k_{1}^{\prime}+k_{2}^{\prime}\right)}{2}\right\rfloor 。$

把上述各式相加, 可得:

当 $x=1, p=2, k_{1}^{\prime}+k_{2}^{\prime}=0 \quad$ 时

$W\left(G_{4}\right)-W\left(G_{4}^{\prime}\right)=0$, 即 $G_{4} \cong C_{5}$; 当 $x=1$ 且 $p>2$ 或 $x>1$ 时, $W\left(G_{4}\right)-W\left(G_{4}^{\prime}\right)>0$ 。

综上所述: $W\left(G_{4}\right) \geq W\left(G_{4}^{\prime}\right)$, 等式成立当且仅当 $k_{1}^{\prime}+k_{2}^{\prime}=0$ 且 $U_{2}$ 的圈长为 4 或者 $G_{4} \cong C_{5}$ 。

证毕。

不妨设图 $G_{4}$ 中有 $f$ 个圈长度大于 3 , 则对图 $G_{4}$ 运用 变换 $C$ 共 $f$ 次, 得到图 $G_{5}$ (见图 11 ) , 记为 $C^{(f)}\left(G_{4}\right)=G_{5}$ 。

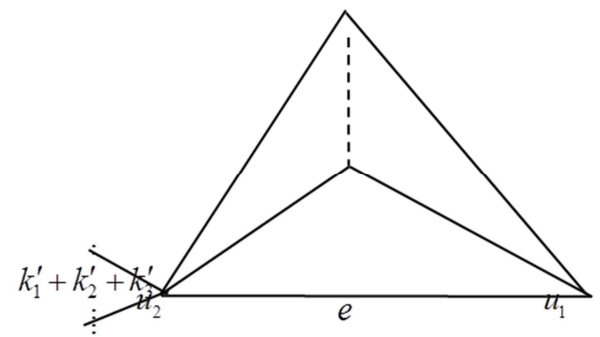

图11书图 $G_{5}$ 。

由性质2.4可得:

引理2. 4 设 $C^{(f)}\left(G_{4}\right)=G_{5}$, 则 $W\left(G_{4}\right) \geq W\left(G_{5}\right)$, 等式成立当且仅当 $G_{4} \cong G_{5}$ 或者 $f=1, k_{1}^{\prime}+k_{2}^{\prime}=0$,

$U_{2}$ 的圈长为 4 或者 $G_{4} \cong C_{5}$ 。

引理 $2.5 W\left(G_{5}\right) \geq W\left(G_{6}\right)$, 等式成立当且仅当 $G_{5} \cong G_{6}$ （见图12）。

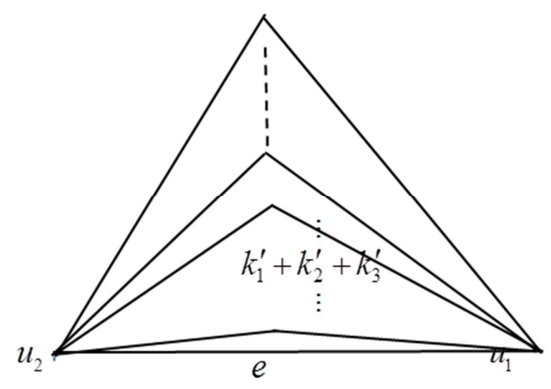

图12书图 $G_{6}$ 。
证明图 $G_{5}$ 与 $G_{6}$ 中只有悬挂点与点 $u_{1}$ 的距离由二变 一, 其它点间距离没有变化, 故 $W\left(G_{5}\right)-W\left(G_{6}\right)=k_{1}^{\prime}+k_{2}^{\prime}+k_{3}^{\prime} \geq 0$, 等式成立当且仅当 $k_{1}^{\prime}+k_{2}^{\prime}+k_{3}^{\prime}=0$, 即 $G_{5} \cong G_{6}$ 。证毕。

引理2. $6 W\left(C_{5}\right)>W\left(G_{6}\right), W\left(\widetilde{U}_{2}\right)>W\left(G_{6}\right)$, 其中 $\widetilde{U}_{2}$ 表示引理 2.4 中 $f=1, k_{1}^{\prime}+k_{2}^{\prime}=0, U_{2}$ 的圈长 为 4 的图。

证 明 $W\left(C_{5}\right)=15>W\left(\left.G_{6}\right|_{n=5}\right)=13$, $W\left(\left.\widetilde{U}_{2}\right|_{n=4}\right)=8>W\left(\left.G_{6}\right|_{n=4}\right)=7, \begin{aligned} & W\left(\left.\tilde{U}_{2}\right|_{n>4}\right)=n^{2}-3 n+4 \\ & >W\left(\left.G_{6}\right|_{n>4}\right)=n^{2}-3 n+3\end{aligned} 。$ 证毕。

定理 设 $G_{1}$ 为 $n$ 阶书图, 则 $W\left(G_{1}\right) \geq n^{2}-3 n+3$, 等式成立当且仅当 $G_{1} \cong G_{6}$

证明首先用数学归纳法证明 $W\left(G_{6}\right)=n^{2}-3 n+3$ :

当 $n=3$ 时, $W\left(G_{6}\right)=3$ 。

假 设 当 $n=k$ 时, 等式 成 立, 即 $W\left(G_{6}\right)=k^{2}-3 k+3$ 。

当 $n=k+1$ 时,

$$
=(k+1)^{2}-3(k+1)+3
$$

由引理 2. 1-2.6知, $W\left(G_{1}\right) \geq W\left(G_{6}\right)$, 等式成立当 且仅当 $G_{1} \cong G_{6}$ 。

故 $W\left(G_{1}\right) \geq n^{2}-3 n+3$, 等式成立当且仅当 $G_{1} \cong G_{6}$ 。证毕。

\section{3. 结论}

本文运用图论的方法, 定义了三种变换; 证明了这三 种变换后图的维纳指数单调下降; 给出了六个引理的数学 证明; 得到了书图的维纳指数的最小值, 并且确定了维纳 指数达到最小的极图。这个极图可以用于化学分子的定量 结构性质和活性关系的研究中。

\section{致谢}

本文由2015年国家级大学生创新创业项目资助, 编号: 201510564282 。

\section{参考文献}

[1] Chen Ya-hong. The Wiener Index of Unicyclic Graphs with Perfect Matching [J]. Journal of Shanghai Jiaotong University, 2010. 
[2] Hosoya H. Topologicalindex. A newly proposed quantity characterizing the topological nature of struc-tural isomers of saturated hydrocarbons $[\mathrm{J}]$. Bull Chem Soc Japan, 1971, 44(9) : 2332-2339.

[3] Plesnik J. On the sum of all distances in a graph or digraph [J]. J Graph Theory, 1984, 8(1) : 1-12.

[4] Wiener H. Structral determination of paraffin boiling points [J]. J Am Chem Soc, 1947, 69(1): 17-20.

[5] GutmanI, KlavzarS, MoharB. Fifty years of the Wiener index [J]. MATCH Commun Math Comput hem, 1997, 35 (1) : $1-159$.

[6] Gutman I , Klavzar S, Mohar B. Fiftieth anniversary of the Wiener index [J]. Discrete Appl Math, 1997, 80 (1): $1-113$.
[7] Jiao Shijie, Zhou Bo, On the spectral radius of books [D]. South China Normal University. 2015.

[8] R. Todeschini, V. Consonni, Handbook of Molecular Descriptors, Wiley-VCH, Weinheim, 2000.

[9] Xiaotie Deng Jie Zhang, Equiseparability on terminal Wiener index, Applied Mathematics Letters 25(2012): $580^{--585 .}$

[10] Liu Wu-xin, Wei Fu-yi\#, On the incidence Estrada index of graphs, Util. Math. 2015, 98: 43-52.

[11] The Variable Wiener Index of Trees with Given Maximum Degree, Communications and Information Technology, 16-17, 2014, P36-37. 